\title{
Stability Analysis of Impulsive Stochastic Cohen-Grossberg Neural Networks with Mixed Time Delays
}

\author{
Qiankun Song $^{a}$ and Zidong Wang,*
}

\begin{abstract}
In this paper, the problem of stability analysis for a class of impulsive stochastic Cohen-Grossberg neural networks with mixed delays is considered. The mixed time delays comprise both the time-varying and infinite distributed delays. By employing a combination of the $M$-matrix theory and stochastic analysis technique, a sufficient condition is obtained to ensure the existence, uniqueness, and exponential $p$-stability of the equilibrium point for the addressed impulsive stochastic Cohen-Grossberg neural network with mixed delays. The proposed method, which does not make use of the Lyapunov functional, is shown to be simple yet effective for analyzing the stability of impulsive or stochastic neural networks with variable and/or distributed delays. We then extend our main results to the case where the parameters contain interval uncertainties. Moreover, the exponential convergence rate index is estimated, which depends on the system parameters. An example is given to show the effectiveness of the obtained results.
\end{abstract}

\section{Keywords}

Cohen-Grossberg neural networks; Stochastic neural networks; Exponential p-stability; Time-varying delays; Distributed delays; Impulsive effect.

\section{INTRODUCTION}

The Cohen-Grossberg neural network model, first proposed and studied by Cohen and Grossberg in 1983 [1], has attracted considerable attention due to its potential applications in classification, parallel computing, associative memory, signal and image processing, especially in solving some difficult optimization problems. In such applications, it is of prime importance to ensure that the designed neural networks be stable [2]. In practice, due to the finite speeds of the switching and transmission of signals, time delays do exist in a working network and thus should be incorporated into the model equation [3,26,27]. In recent years, the dynamical behaviors of Cohen-Grossberg neural networks with constant delays or time-varying delays or distributed delays have been studied, see for example [3-13] and the references therein.

Impulsive effect is likely to exist in a wide variety of evolutionary processes in which states are changed abruptly at certain moments of time in the fields such as medicine and biology, economics, electronics and telecommunications. Neural networks, which include Hopfield neural networks, cellular neural networks and Cohen-Grossberg neural networks, are often subject to impulsive perturbations that in turn affect dynamical

This work was supported by the Natural Science Foundation of CQ CSTC under grant 2007BB0430, the Scientific Research Fund of Chongqing Municipal Education Commission under Grant KJ070401, an International Joint Project sponsored by the Royal Society of the U.K. and the National Natural Science Foundation of China, and the Alexander von Humboldt Foundation of Germany.

${ }^{a}$ Department of Mathematics, Chongqing Jiaotong University, Chongqing 400074, China. Email: qiankunsong@163.com

${ }^{b}$ Department of Information Systems and Computing, Brunel University, Uxbridge, Middlesex, UB8 3PH, United Kingdom. Email: Zidong.Wang@brunel.ac.uk, Fax: ++44/1895 251686.

${ }^{*}$ Corresponding author. 
behaviors of the systems. Therefore, it is necessary to consider both the impulsive effect and delay effect when investigating the stability of neural networks [14]. So far, several interesting results have been reported that have focused on the impulsive effect of delayed neural networks, see [14-20] for some recent publications.

In addition to the delay and impulsive effects, stochastic effects constitute another source of disturbances or uncertainties in real systems $[21,31,32]$. A lot of dynamical systems have variable structures subject to stochastic abrupt changes, which may result from abrupt phenomena such as stochastic failures and repairs of the components, changes in the interconnections of subsystems or sudden environment switching [22]. Therefore, stochastic perturbations should be taken into account when modeling neural networks. In recent years, the dynamic analysis of stochastic systems (including neural networks) with delays has been an attractive topic for many researchers, and a large number of stability criteria of these systems have been reported, see e.g. [21-25, 28-30,33,34] and the references therein. In particular, in [21,22], the authors have considered the exponential $p$-stability of impulsive stochastic differential equations with constant delays and obtained several stability conditions for checking the exponential $p$-stability. In $[24,25,28,29,33,34]$, the stability of stochastic neural networks with constant or time-varying delay or bounded distributed delays have been considered and many interesting results have been established by employing a Lyapunov functional approach. To the best of our knowledge, so far, few authors have considered the problem of stability analysis for Cohen-Grossberg neural networks with both time-varying and infinite distributed delays in the simultaneous presence of the impulsive and stochastic effects.

Since both the impulsive and stochastic effects exist in the model, it becomes mathematically complicate to investigate the stability of impulsive stochastic Cohen-Grossberg neural networks with both time-varying and infinite distributed delays. Many existing stability criteria for impulsive Cohen-Grossberg neural networks [20] and stochastic Cohen-Grossberg neural networks [24,29] may be difficult to be applied or even ineffective in dealing with the addressed impulsive stochastic Cohen-Grossberg neural networks. In this case, new techniques will have to be developed. In this paper, we present a novel approach that employs a combination of the $M$-matrix theory and stochastic analysis technique. Using this approach, we give a sufficient condition ensuring the existence, uniqueness, and exponential $p$-stability of equilibrium point for impulsive stochastic Cohen-Grossberg neural networks with time-varying delays and infinite distributed delays. We then extend our main results to the case where the parameters contain interval uncertainties. Moreover, the exponential convergence rate index is estimated which depends on the system parameters, and an example is given to show the effectiveness of the obtained results.

\section{MODEL DESCRIPTION AND PRELIMINARIES}

In this paper, we consider the following model

$$
\left\{\begin{aligned}
d u_{i}(t)=-a_{i}\left(u_{i}(t)\right)\left[b_{i}\left(u_{i}(t)\right)-\sum_{j=1}^{n} c_{i j} g_{j}\left(u_{j}(t)\right)-\sum_{j=1}^{n} d_{i j} f_{j}\left(u_{j}\left(t-\tau_{i j}(t)\right)\right)\right. & \\
& \left.-\sum_{j=1}^{n} v_{i j} \int_{-\infty}^{t} K_{i j}(t-s) h_{j}\left(u_{j}(s)\right) d s+I_{i}\right] d t \\
& +\sum_{j=1}^{n} \sigma_{i j}\left(u_{j}(t), u_{j}\left(t-\tau_{i j}(t)\right)\right) d \omega_{j}(t), \quad t \neq t_{k},
\end{aligned}\right.
$$

for $i=1,2, \cdots, n$ and $k=1,2, \cdots$, where $n$ corresponds to the number of units in the neural network; $u_{i}(t)$ corresponds to the state of the $i$ th unit at time $t$. The first part is the continuous part of model (1), which describes the continuous evolution processes of the neural network, where $g_{j}, f_{j}$ and $h_{j}$ denote the activation 
functions; $\tau_{i j}(t)$ corresponds to the transmission delay along the axon of the $j$ th unit from the $i$ th unit and satisfies $0 \leq \tau_{i j}(t) \leq \tau_{i j}\left(\tau_{i j}\right.$ is a constant); $a_{i}\left(u_{i}(t)\right)$ represents an amplification function at time $t ; b_{i}\left(u_{i}(t)\right)$ is an appropriately behaved function at time $t$ such that the solutions of model (1) remain bounded; $C=\left(c_{i j}\right)_{n \times n}$, $D=\left(d_{i j}\right)_{n \times n}$ and $V=\left(v_{i j}\right)_{n \times n}$ are connection matrices; $K_{i j}$ is the delay kernel function; $I_{i}$ is the constant input from outside of the network; $\sigma_{i j}\left(u_{j}(t), u_{j}\left(t-\tau_{i j}(t)\right)\right)$ is the diffusion coefficient, $\sigma_{i}=\left(\sigma_{i 1}, \sigma_{i 2}, \cdots, \sigma_{i n}\right)$; $\omega(t)=\left(\omega_{1}(t), \omega_{2}(t), \cdots, \omega_{n}(t)\right)^{T}$ is an $n$-dimensional Brownian motion defined on a complete probability space $\left(\Omega, F,\left\{F_{t}\right\}_{t \geq 0}, P\right)$ with a filtration $\left\{F_{t}\right\}_{t \geq 0}$ satisfying the usual conditions (i.e., it is right continuous and $F_{0}$ contains all $P$-null sets). The second part is the discrete part of model (1), which describes that the evolution processes experience abrupt change of state at the moments of time $t_{k}$ (called impulsive moments), where $\Delta u_{i}\left(t_{k}\right)=u_{i}\left(t_{k}^{+}\right)-u_{i}\left(t_{k}^{-}\right)$is the impulses at moment $t_{k}$, the fixed moments of time $t_{k}$ satisfy $t_{1}<t_{2}<\cdots$, $\lim _{k \rightarrow+\infty} t_{k}=+\infty$ and $\min _{2 \leq k<\infty}\left\{t_{k}-t_{k-1}\right\}>\max _{1 \leq i, j \leq n}\left\{\tau_{i j}\right\}$.

Remark 1. Model (1) includes the following impulsive Cohen-Grossberg neural network model as a special case:

$$
\left\{\begin{array}{c}
\frac{d u_{i}(t)}{d t}=-a_{i}\left(u_{i}(t)\right)\left[b_{i}\left(u_{i}(t)\right)-\sum_{j=1}^{n} c_{i j} g_{j}\left(u_{j}(t)\right)-\sum_{j=1}^{n} d_{i j} f_{j}\left(u_{j}\left(t-\tau_{i j}(t)\right)\right)\right. \\
\left.-\sum_{j=1}^{n} v_{i j} \int_{-\infty}^{t} K_{i j}(t-s) h_{j}\left(u_{j}(s)\right) d s+I_{i}\right], \quad t \neq t_{k} \\
\Delta u_{i}\left(t_{k}\right)=J_{k}\left(u_{i}\left(t_{k}^{-}\right)\right)
\end{array}\right.
$$

for $i=1,2, \cdots, n$ and $k=1,2, \cdots$.

When $J_{k}\left(u_{i}\left(t_{k}\right)\right)=0(i=1,2, \cdots, n ; k=1,2, \cdots)$, model (1) turns into the following stochastic CohenGrossberg neural network model without impulses:

$$
\begin{aligned}
d u_{i}(t)= & -a_{i}\left(u_{i}(t)\right)\left[b_{i}\left(u_{i}(t)\right)-\sum_{j=1}^{n} c_{i j} g_{j}\left(u_{j}(t)\right)-\sum_{j=1}^{n} d_{i j} f_{j}\left(u_{j}\left(t-\tau_{i j}(t)\right)\right)\right. \\
& \left.-\sum_{j=1}^{n} v_{i j} \int_{-\infty}^{t} K_{i j}(t-s) h_{j}\left(u_{j}(s)\right) d s+I_{i}\right] d t+\sum_{j=1}^{n} \sigma_{i j}\left(u_{j}(t), u_{j}\left(t-\tau_{i j}(t)\right)\right) d \omega_{j}(t)
\end{aligned}
$$

for $t>0, \quad i=1,2, \cdots, n$. Furthermore, model (3) also comprises the following Cohen-Grossberg neural network model with neither impulses nor stochastic effects [13]

$$
\begin{aligned}
\frac{d u_{i}(t)}{d t}= & -a_{i}\left(u_{i}(t)\right)\left[b_{i}\left(u_{i}(t)\right)-\sum_{j=1}^{n} c_{i j} g_{j}\left(u_{j}(t)\right)-\sum_{j=1}^{n} d_{i j} f_{j}\left(u_{j}\left(t-\tau_{i j}(t)\right)\right)\right. \\
& \left.-\sum_{j=1}^{n} v_{i j} \int_{-\infty}^{t} K_{i j}(t-s) h_{j}\left(u_{j}(s)\right) d s+I_{i}\right]
\end{aligned}
$$

for $t>0, \quad i=1,2, \cdots, n$. Note that model (4) is also a general neural network that covers the delayed Cohen-Grossberg neural network models studied in [3-12].

Since the solution $\left(u_{1}(t), u_{2}(t), \cdots, u_{n}(t)\right)^{T}$ of model (2) is discontinuous at the point $t_{k}$, by theory of impulsive differential equations, we assume that $\left(u_{1}\left(t_{k}\right), u_{2}\left(t_{k}\right), \cdots, u_{n}\left(t_{k}\right)\right) \equiv\left(u_{1}\left(t_{k}+0\right), u_{2}\left(t_{k}+0\right), \cdots, u_{n}\left(t_{k}+0\right)\right)^{T}$. It is clear that, in general, the derivatives $\frac{d u_{i}\left(t_{k}\right)}{d t}$ does not exist. On the other hand, we can see from the first equation of model (2) that the limits $\frac{d u_{i}\left(t_{k} \mp 0\right)}{d t}$ exist. According to the above convention, we assume $\frac{d u_{i}\left(t_{k}\right)}{d t}=\frac{d u_{i}\left(t_{k}+0\right)}{d t}$

For convenience, we introduce several notations. $u=\left(u_{1}, u_{2}, \cdots, u_{n}\right)^{T} \in R^{n}$ denotes a column vector; $\|u\|$ denotes a vector norm defined by $\|u\|=\left(\sum_{j=1}^{n}\left|u_{i}\right|^{p}\right)^{1 / p} \cdot C[X, Y]$ denotes the space of continuous mappings 
from the topological space $X$ to the topological space $Y$. Denote by $C_{F_{0}}^{b}\left[(-\infty, 0], R^{n}\right]$ the family of all bounded $F_{0}$-measurable, $C\left[(-\infty, 0], R^{n}\right]$-valued random variables $\phi$, satisfying $\|\phi\|_{L^{p}}=\sup _{-\infty \leq \theta \leq 0} E\|\phi(\theta)\|<+\infty$, where $E$ denotes the expectation of stochastic process. The initial condition $\phi \in C_{F_{0}}^{b}\left[(-\infty, 0], R^{n}\right] . P C\left[I, R^{n}\right]=\{\psi$ : $I \rightarrow R^{n} \mid \psi\left(t^{+}\right)=\psi(t)$ for $t \in I, \psi\left(t^{-}\right)$exists for $t \in\left(t_{0},+\infty\right), \psi\left(t^{-}\right)=\psi(t)$ for all but points $\left.t_{k} \in\left(t_{0},+\infty\right)\right\}$, where $I \subset R$ is an interval, $\psi\left(t^{+}\right)$and $\psi\left(t^{-}\right)$denote the left-hand limit and right-hand limit of the scalar function $\psi(t)$, respectively.

Throughout this paper, we make the following assumptions:

$(\mathbf{H} 1) a_{i}(u)$ is a continuous function and $0<a_{i} \leq a_{i}(u)<A_{i}\left(a_{i}\right.$ and $A_{i}$ are constants) for all $u \in R$, $i=1,2, \cdots, n$.

(H2) There exists a positive diagonal matrix $B=\operatorname{diag}\left(b_{1}, b_{2}, \cdots, b_{n}\right)$ such that

$$
\frac{b_{i}(u)-b_{i}(v)}{u-v} \geq b_{i}
$$

for all $u, v \in R(u \neq v), i=1,2, \cdots, n$.

(H3) There exist three positive diagonal matrices $G=\operatorname{diag}\left(G_{1}, G_{2}, \cdots, G_{n}\right), F=\operatorname{diag}\left(F_{1}, F_{2}, \cdots, F_{n}\right)$ and $H=\operatorname{diag}\left(H_{1}, H_{2}, \cdots, H_{n}\right)$ such that

$$
G_{j}=\sup _{u_{1} \neq u_{2}}\left|\frac{g_{j}\left(u_{1}\right)-g_{j}\left(u_{2}\right)}{u_{1}-u_{2}}\right|, \quad F_{j}=\sup _{u_{1} \neq u_{2}}\left|\frac{f_{j}\left(u_{1}\right)-f_{j}\left(u_{2}\right)}{u_{1}-u_{2}}\right|, \quad H_{j}=\sup _{u_{1} \neq u_{2}}\left|\frac{h_{j}\left(u_{1}\right)-h_{j}\left(u_{2}\right)}{u_{1}-u_{2}}\right|
$$

for all $u_{1} \neq u_{2}, j=1,2, \cdots, n$.

$(\mathbf{H} 4)$ The delay kernel $K_{i j}:[0,+\infty) \rightarrow[0,+\infty)$ is real valued nonnegative continuous function and satisfies

$$
\int_{0}^{+\infty} e^{\beta s} K_{i j}(s) d s=r_{i j}(\beta)
$$

where $r_{i j}(\beta)$ is continuous function in $[0, \delta), \delta>0$, and $r_{i j}(0)=1, i, j=1,2, \cdots, n$.

$(\mathbf{H} 5)$ There exist two nonnegative matrices $S=\left(s_{i j}\right)_{n \times n}$ and $W=\left(w_{i j}\right)_{n \times n}$ such that

$$
\sigma_{i}(u, v) \sigma_{i}^{T}(u, v) \leq \sum_{j=1}^{n} s_{i j} u_{j}^{2}(t)+\sum_{j=1}^{n} w_{i j} v_{j}^{2}
$$

for all $u=\left(u_{1}, \cdots u_{n}\right)^{T} \in R^{n}, v=\left(v_{1}, \cdots v_{n}\right)^{T} \in R^{n}, i=1,2, \cdots, n$.

Definition 1: The equilibrium point $u^{*}=\left(u_{1}^{*}, u_{2}^{*}, \cdots, u_{n}^{*}\right)^{T}$ of model (1) is said to be globally exponentially $p$-stable $(p \geq 2)$ if there exist constants $\varepsilon>0$ and $M>0$ such that

$$
E\left\|u(t)-u^{*}\right\|^{p} \leq M\left\|\phi-u^{*}\right\|_{L^{p}}^{p} e^{-\varepsilon\left(t-t_{0}\right)}
$$

for all $t>0$, where $u(t)=\left(u_{1}(t), u_{2}(t), \cdots, u_{n}(t)\right)^{T}$ is any solution of model (1) with initial value $u_{i}\left(t_{0}+s\right)=$ $\phi_{i}(s) \in P C((-\infty, 0], R), i=1,2, \cdots, n$.

Definition 2: A real matrix $A=\left(a_{i j}\right)_{n \times n}$ is said to be an $M$-matrix if $a_{i j} \leq 0(i, j=1,2, \cdots, n ; i \neq j)$ and successive principle minors of $A$ are positive.

Definition 3: (Song and Cao [13]) A map $H: \quad R^{n} \rightarrow R^{n}$ is a homeomorphism of $R^{n}$ onto itself, if $H \in C^{0}$, $H$ is one-to-one, $H$ is onto and the inverse map $H^{-1} \in C^{0}$.

To prove our results, the following lemmas that can be found in $[8,13]$ are necessary.

Lemma 1: (Cao and Liang [8]) Let $a, b \geq 0, p \geq i>0$, then

$$
a^{p-i} b^{i} \leq \frac{p-i}{p} a^{p}+\frac{i}{p} b^{p} .
$$


Lemma 2: (Song and Cao [13]) Let $Q$ be $n \times n$ matrix with non-positive off-diagonal elements, then $Q$ is an $M$-matrix if and only if one of the following conditions holds:

(i) There exists a vector $\xi>0$ such that $\xi^{T} Q>0$.

(ii) There exists a vector $\xi>0$ such that $Q \xi>0$.

Lemma 3: (Song and Cao [13]) If $H(x) \in C^{0}$ satisfies the following conditions

(i) $H(x)$ is injective on $R^{n}$,

(ii) $\|H(x)\| \rightarrow+\infty$ as $\|x\| \rightarrow+\infty$,

then $H(x)$ is homeomorphism of $R^{n}$ onto itself.

\section{MAIN RESULTS}

Theorem 1: Under assumptions $(\mathbf{H} 1)-(\mathbf{H} 5)$, if there exists a positive constant $p \geq 2$ such that $-(Q+T)$ is an $M$-matrix, where

$$
\begin{gathered}
Q=\left(q_{i j}\right)_{n \times n}, \quad q_{i j}=\left|c_{i j}\right| G_{j}+\frac{p-1}{A_{i}} s_{i j}, \quad i \neq j, \\
q_{i i}=-p b_{i} \frac{a_{i}}{A_{i}}+(p-1)\left(\sum_{j=1}^{n}\left|c_{i j}\right| G_{j}+\sum_{j=1}^{n}\left|d_{i j}\right| F_{j}+\sum_{j=1}^{n}\left|v_{i j}\right| H_{j}\right. \\
\left.+\frac{p-2}{2 A_{i}} \sum_{j=1}^{n} s_{i j}+\frac{p-2}{2 A_{i}} \sum_{j=1}^{n} w_{i j}\right)+\left|c_{i i}\right| G_{i}+\frac{p-1}{A_{i}} s_{i i}, \\
T=\left(t_{i j}\right)_{n \times n}, \quad t_{i j}=\left|d_{i j}\right| F_{j}+\frac{p-1}{A_{i}} w_{i j}+\left|v_{i j}\right| H_{j},
\end{gathered}
$$

then model (4) has a unique equilibrium point $\left(u_{1}^{*}, u_{2}^{*}, \cdots, u_{n}^{*}\right)^{T}$. Furthermore, suppose

(i) $\sigma_{i j}\left(u_{j}^{*}, u_{j}^{*}\right)=0, i, j=1,2, \cdots, n$;

(ii) $J_{k}\left(u_{i}\left(t_{k}\right)\right)=-\gamma_{i k}\left(u_{i}\left(t_{k}^{-}\right)-u_{i}^{*}\right), \quad 0<\gamma_{i k}<2, \quad i=1,2, \cdots, n ; \quad k=1,2, \cdots$.

Then, $\left(u_{1}^{*}, u_{2}^{*}, \cdots, u_{n}^{*}\right)^{T}$ is a unique globally exponentially $p$-stable equilibrium point of model (1).

Proof. We shall prove this theorem in two steps.

Step 1: We will prove the existence and uniqueness of the equilibrium point of model (4) under the given assumptions.

Let $H(x)=\left(H_{1}(x), H_{2}(x), \cdots, H_{n}(x)\right)^{T}$, where

$$
H_{i}(x)=-b_{i}\left(x_{i}\right)+\sum_{j=1}^{n} c_{i j} g_{j}\left(x_{j}\right)+\sum_{j=1}^{n} d_{i j} f_{j}\left(x_{j}\right)+\sum_{j=1}^{n} v_{i j} h_{j}\left(x_{j}\right)-I_{i}
$$

for $i=1,2, \cdots, n$. In the following, we shall prove that $H(x)$ is a homeomorphism of $R^{n}$ onto itself.

First, we prove that $H(x)$ is an injective map on $R^{n}$. In fact, if there exist $x=\left(x_{1}, x_{2}, \cdots, x_{n}\right)^{T}$ and $y=\left(y_{1}, y_{2}, \cdots, y_{n}\right)^{T} \in R^{n}$ and $x \neq y$ such that $H(x)=H(y)$, then

$$
b_{i}\left(x_{i}\right)-b_{i}\left(y_{i}\right)=\sum_{j=1}^{n} c_{i j}\left(g_{j}\left(x_{j}\right)-g_{j}\left(y_{j}\right)\right)+\sum_{j=1}^{n} d_{i j}\left(f_{j}\left(x_{j}\right)-f_{j}\left(y_{j}\right)\right)+\sum_{j=1}^{n} v_{i j}\left(h_{j}\left(x_{j}\right)-h_{j}\left(y_{j}\right)\right)
$$

for $i=1,2, \cdots, n$. Multiply both sides of (5) by $\left|x_{i}-y_{i}\right|^{p-1}$, it follows from assumptions $(\mathbf{H} 2),(\mathbf{H} 3)$ and Lemma 1 that

$$
\left(p b_{i}-(p-1) \sum_{j=1}^{n}\left(\left|c_{i j}\right| G_{j}+\left|d_{i j}\right| F_{j}+\left|v_{i j}\right| H_{j}\right)\left|x_{i}-y_{i}\right|^{p} \leq \sum_{j=1}^{n}\left(\left|c_{i j}\right| G_{j}+\left|d_{i j}\right| F_{j}+\left|v_{i j}\right| H_{j}\right)\left|x_{j}-y_{j}\right|^{p}\right.
$$


for $i=1,2, \cdots, n$. Let $\Upsilon=\left(\alpha_{i j}\right)_{n \times n}$, where

$$
\begin{gathered}
\alpha_{i i}=p b_{i}-(p-1) \sum_{j=1}^{n}\left(\left|c_{i j}\right| G_{j}+\left|d_{i j}\right| F_{j}+\left|v_{i j}\right| H_{j}\right)-\left|c_{i i}\right| G_{i}-\left|d_{i i}\right| F_{i}-\left|v_{i i}\right| H_{i}, \quad i=1,2, \cdots, n, \\
\alpha_{i j}=-\left|c_{i j}\right| G_{j}-\left|d_{i j}\right| F_{j}-\left|v_{i j}\right| H_{j}, \quad i \neq j, i, j=1,2, \cdots, n .
\end{gathered}
$$

Then (6) becomes the following

$$
\Upsilon\left(\left|x_{1}-y_{1}\right|^{p},\left|x_{2}-y_{2}\right|^{p}, \cdots,\left|x_{n}-y_{n}\right|^{p}\right)^{T} \leq 0 .
$$

Let $-(Q+T)=\left(\beta_{i j}\right)_{n \times n}$. Noting $\frac{a_{i}}{A_{i}} \leq 1$, and $s_{i j}, w_{i j} \geq 0$ and $p \geq 2$, we can get that

$$
\beta_{i j} \leq \alpha_{i j}, \quad i, j=1,2 \cdots, n .
$$

Since $-(Q+T)$ is an $M$-matrix and $\Upsilon$ is a matrix with non-positive off-diagonal elements, $\Upsilon$ is also an $M$-matrix. It follows from (7) that

$$
x_{i}=y_{i}, \quad i=1,2, \cdots, n,
$$

which is a contradiction, so $H(x)$ is an injective on $R^{n}$.

Next, we prove that $\|H(x)\| \rightarrow+\infty$ as $\|x\| \rightarrow+\infty$. Since $\Upsilon$ is an $M$-matrix, from (i) of Lemma 2 , there exists a positive vector $\xi=\left(\xi_{1}, \xi_{2}, \cdots, \xi_{n}\right)^{T}$ such that

$$
\xi_{i}\left(p b_{i}-(p-1) \sum_{j=1}^{n}\left(\left|c_{i j}\right| G_{j}+\left|d_{i j}\right| F_{j}+\left|v_{i j}\right| H_{j}\right)\right)-\sum_{j=1}^{n} \xi_{j}\left(\left|c_{j i}\right| G_{i}+\left|d_{j i}\right| F_{i}+\left|v_{j i}\right| H_{i}\right)>0
$$

for $i=1,2, \cdots, n$. We can choose a small number $\delta>0$ such that

$$
\xi_{i}\left(p b_{i}-(p-1) \sum_{j=1}^{n}\left(\left|c_{i j}\right| G_{j}+\left|d_{i j}\right| F_{j}+\left|v_{i j}\right| H_{j}\right)\right)-\sum_{j=1}^{n} \xi_{j}\left(\left|c_{j i}\right| G_{i}+\left|d_{j i}\right| F_{i}+\left|v_{j i}\right| H_{i}\right) \geq \delta>0
$$

for $i=1,2, \cdots, n$. Let

$$
\widetilde{H}(x)=\left(\widetilde{H}_{1}(x), \widetilde{H}_{2}(x), \cdots, \widetilde{H}_{n}(x)\right)^{T},
$$

where

$$
\widetilde{H}_{i}(x)=-\left(b_{i}\left(x_{i}\right)-b_{i}(0)\right)+\sum_{j=1}^{n} c_{i j}\left(g_{j}\left(x_{j}\right)-g_{j}(0)\right)+\sum_{j=1}^{n} d_{i j}\left(f_{j}\left(x_{j}\right)-f_{j}(0)\right)+\sum_{j=1}^{n} v_{i j}\left(h_{j}\left(x_{j}\right)-h_{j}(0)\right)
$$

for $i=1,2, \cdots, n$. From assumptions $(\mathbf{H} 2),(\mathbf{H} 3)$ and Lemma 1, we can get

$$
\begin{aligned}
\sum_{i=1}^{n} p \xi_{i}\left|x_{i}\right|^{p-1} \operatorname{sgn}\left(x_{i}\right) \tilde{H}_{i}(x) \leq & \sum_{i=1}^{n}\left[\xi_{i}\left(-p b_{i}+(p-1) \sum_{j=1}^{n}\left(\left|c_{i j}\right| G_{j}+\left|d_{i j}\right| F_{j}+\left|v_{i j}\right| H_{j}\right)\right)\right. \\
& \left.+\sum_{j=1}^{n} \xi_{j}\left(\left|c_{j i}\right| G_{i}+\left|d_{j i}\right| F_{i}+\left|v_{j i}\right| H_{i}\right)\right]\left|x_{i}\right|^{p} \\
\leq & -\delta\|x\|^{p} .
\end{aligned}
$$

Thus

$$
\delta\|x\|^{p} \leq \sum_{i=1}^{n} p \xi_{i}\left|x_{i}\right|^{p-1}\left|\tilde{H}_{i}(x)\right| \leq p \max _{1 \leq i \leq n}\left\{\xi_{i}\right\} \sum_{i=1}^{n}\left|x_{i}\right|^{p-1}\left|\tilde{H}_{i}(x)\right| .
$$


By using Hölder inequality, we get

$$
\delta\|x\|^{p} \leq p \max _{1 \leq i \leq n}\left\{\xi_{i}\right\}\|x\|^{p-1}\left\|\tilde{H}_{i}(x)\right\|
$$

that is

$$
\delta\|x\| \leq p \max _{1 \leq i \leq n}\left\{\xi_{i}\right\}\|\tilde{H}(x, y)\| .
$$

Therefore $\|\widetilde{H}(x)\|_{\infty} \rightarrow+\infty$ as $\|(x)\|_{\infty} \rightarrow+\infty$, which directly implies that $\|H(x)\| \rightarrow+\infty$ as $\|(x)\| \rightarrow+\infty$

By Lemma 3, we know that $H(x)$ is a homeomorphism on $R^{n}$. Thus equation

$$
-b_{i}\left(x_{i}\right)+\sum_{j=1}^{n} c_{i j} g_{j}\left(x_{j}\right)+\sum_{j=1}^{n} d_{i j} f_{j}\left(x_{j}\right)+\sum_{j=1}^{n} v_{i j} h_{j}\left(x_{j}\right)-I_{i}=0, \quad i=1,2, \cdots, n
$$

has unique solution $\left(u_{1}^{*}, u_{2}^{*}, \cdots, u_{n}^{*}\right)^{T}$, which is a unique equilibrium point of model (4) due to assumptions (H1) and (H4).

From conditions (i) and (ii) of this theorem, we know that $\left(u_{1}^{*}, u_{2}^{*}, \cdots, u_{n}^{*}\right)^{T}$ is also a unique equilibrium point of model (1).

Step 2: We prove that the unique equilibrium point $\left(u_{1}^{*}, u_{2}^{*}, \cdots, u_{n}^{*}\right)^{T}$ of model $(1)$ is globally exponentially $p$-stable.

By denoting

$$
\begin{gathered}
y_{i}(t)=u_{i}(t)-u_{i}^{*}, \quad \widetilde{a}_{i}\left(y_{i}(t)\right)=a_{i}\left(y_{i}(t)+u_{i}^{*}\right), \quad \widetilde{b}_{i}\left(y_{i}(t)\right)=b_{i}\left(y_{i}(t)+u_{i}^{*}\right)-b_{i}\left(u_{i}^{*}\right), \\
\widetilde{g}_{j}\left(y_{j}(t)\right)=g_{j}\left(y_{j}(t)+u_{j}^{*}\right)-g_{j}\left(u_{j}^{*}\right), \quad \widetilde{f}_{j}\left(y_{j}(t)\right)=f_{j}\left(y_{j}(t)+u_{j}^{*}\right)-f_{j}\left(u_{j}^{*}\right), \\
\widetilde{h}_{j}\left(y_{j}(t)\right)=h_{j}\left(y_{j}(t)+u_{j}^{*}\right)-h_{j}\left(u_{j}^{*}\right), \quad \widetilde{\sigma}_{i j}\left(y_{j}(t)\right)=\sigma_{i j}\left(y_{j}(t)+u_{j}^{*}\right)-\sigma_{i j}\left(u_{j}^{*}\right),
\end{gathered}
$$

we have

$$
\begin{aligned}
d y_{i}(t)= & -\widetilde{a}_{i}\left(y_{i}(t)\right)\left[\widetilde{b}_{i}\left(y_{i}(t)\right)-\sum_{j=1}^{n} c_{i j} \widetilde{g}_{j}\left(y_{j}(t)\right)-\sum_{j=1}^{n} d_{i j} \widetilde{f}_{j}\left(y_{j}\left(t-\tau_{i j}(t)\right)\right)\right. \\
& \left.-\sum_{j=1}^{n} v_{i j} \int_{-\infty}^{t} K_{i j}(t-s) \widetilde{h}_{j}\left(y_{j}(s)\right) d s\right] d t+\sum_{j=1}^{n} \widetilde{\sigma}_{i j}\left(u_{j}(t), u_{j}\left(t-\tau_{i j}(t)\right)\right) d \omega_{j}(t), \quad t \neq t_{k},
\end{aligned}
$$

for $i=1,2, \cdots, n ; k=1,2, \cdots$.

Since $-(Q+T)$ is an $M$-matrix, from (ii) of lemma 2 , there exists $\xi=\left(\xi_{1}, \xi_{2}, \cdots, \xi_{n}\right)^{T}>0$ such that $0<-(Q+T) \xi$, that is

$$
\begin{aligned}
0< & {\left[p b_{i} \frac{a_{i}}{A_{i}}-(p-1)\left(\sum_{j=1}^{n}\left|c_{i j}\right| G_{j}+\sum_{j=1}^{n}\left|d_{i j}\right| F_{j}+\sum_{j=1}^{n}\left|v_{i j}\right| H_{j}\right.\right.} \\
& \left.\left.+\frac{p-2}{2 A_{i}} \sum_{j=1}^{n} s_{i j}+\frac{p-2}{2 A_{i}} \sum_{j=1}^{n} w_{i j}\right)\right] \xi_{i}-\sum_{j=1}^{n}\left[\left(\left|c_{i j}\right| G_{j}+\frac{p-1}{A_{i}} s_{i j}\right)\right. \\
& \left.+\left|d_{i j}\right| F_{j}+\frac{p-1}{A_{i}} w_{i j}+\left|v_{i j}\right| H_{j}\right] \xi_{j}, \quad i=1,2, \cdots, n .
\end{aligned}
$$


We can choose a sufficiently small positive constant $\varepsilon>0$ such that

$$
\begin{aligned}
0< & {\left[p b_{i} \frac{a_{i}}{A_{i}}-\frac{\varepsilon}{A_{i}}-(p-1)\left(\sum_{j=1}^{n}\left|c_{i j}\right| G_{j}+\sum_{j=1}^{n}\left|d_{i j}\right| F_{j}+\sum_{j=1}^{n}\left|v_{i j}\right| H_{j}\right.\right.} \\
& \left.\left.+\frac{p-2}{2 A_{i}} \sum_{j=1}^{n} s_{i j}+\frac{p-2}{2 A_{i}} \sum_{j=1}^{n} w_{i j}\right)\right] \xi_{i}-\sum_{j=1}^{n}\left[\left(\left|c_{i j}\right| G_{j}+\frac{p-1}{A_{i}} s_{i j}\right)\right. \\
& \left.+e^{\varepsilon \tau}\left(\left|d_{i j}\right| F_{j}+\frac{p-1}{A_{i}} w_{i j}\right)+\left|v_{i j}\right| H_{j} r_{i j}(\varepsilon)\right] \xi_{j}, \quad i=1,2, \cdots, n .
\end{aligned}
$$

Let

$$
x_{i}(t)=e^{\varepsilon\left(t-t_{0}\right)}\left|y_{i}(t)\right|^{p}, \quad p \geq 2, \quad i=1,2, \cdots, n .
$$

By Itô differential formula, the stochastic derivative of $x_{i}(t)$ along (9) can be obtained as follows:

$$
\begin{aligned}
L x_{i}(t)= & \varepsilon e^{\varepsilon\left(t-t_{0}\right)}\left|y_{i}(t)\right|^{p}+p e^{\varepsilon\left(t-t_{0}\right)}\left|y_{i}(t)\right|^{p-1} \operatorname{sgn}\left(y_{i}(t)\right)\left\{-\widetilde{a}_{i}\left(y_{i}(t)\right)\left[\widetilde{b}_{i}\left(y_{i}(t)\right)-\sum_{j=1}^{n} c_{i j} \widetilde{g}_{j}\left(y_{j}(t)\right)\right.\right. \\
& \left.\left.-\sum_{j=1}^{n} d_{i j} \widetilde{f}_{j}\left(y_{j}\left(t-\tau_{i j}(t)\right)\right)-\sum_{j=1}^{n} v_{i j} \int_{-\infty}^{t} K_{i j}(t-s) \widetilde{h}_{j}\left(y_{j}(s)\right) d s\right]\right\} \\
& +\frac{1}{2} p(p-1) e^{\varepsilon\left(t-t_{0}\right)}\left|y_{i}(t)\right|^{p-2} \widetilde{\sigma}_{i} \widetilde{\sigma}_{i}^{T}
\end{aligned}
$$

for $i=1,2, \cdots, n ; t_{k-1}<t<t_{k}, k=1,2, \cdots$.

By applying assumptions (H1)-(H3) and (H5), we get

$$
\begin{aligned}
L x_{i}(t) \leq & \varepsilon e^{\varepsilon\left(t-t_{0}\right)}\left|y_{i}(t)\right|^{p}+p e^{\varepsilon\left(t-t_{0}\right)}\left|y_{i}(t)\right|^{p-1}\left[-a_{i} b_{i}\left|y_{i}(t)\right|+A_{i} \sum_{j=1}^{n}\left|c_{i j}\right| G_{j}\left|y_{j}(t)\right|\right. \\
& \left.+A_{i} \sum_{j=1}^{n}\left|d_{i j}\right| F_{j}\left|y_{j}\left(t-\tau_{i j}(t)\right)\right|+A_{i} \sum_{j=1}^{n}\left|v_{i j}\right| \int_{-\infty}^{t} K_{i j}(t-s)\left|y_{j}(s)\right| H_{j} d s\right] \\
& +\frac{1}{2} p(p-1) e^{\varepsilon\left(t-t_{0}\right)}\left|y_{i}(t)\right|^{p-2}\left[\sum_{j=1}^{n} s_{i j} y_{j}^{2}(t)+\sum_{j=1}^{n} w_{i j} y_{j}^{2}\left(t-\tau_{i j}(t)\right)\right]
\end{aligned}
$$


for $i=1,2, \cdots, n ; t_{k-1}<t<t_{k}, k=1,2, \cdots$. It follows from Lemma 1 that

$$
\begin{aligned}
L x_{i}(t) \leq & \varepsilon x_{i}(t)-p a_{i} b_{i} x_{i}(t)+A_{i}\left[(p-1) \sum_{j=1}^{n}\left|c_{i j}\right| G_{j} x_{i}(t)+\sum_{j=1}^{n}\left|c_{i j}\right| G_{j} x_{j}(t)\right. \\
& +(p-1) \sum_{j=1}^{n}\left|d_{i j}\right| F_{j} x_{i}(t)+\sum_{j=1}^{n}\left|d_{i j}\right| F_{j} e^{\varepsilon \tau_{i j}(t)} x_{j}\left(t-\tau_{i j}(t)\right) \\
& \left.+(p-1) \sum_{j=1}^{n}\left|v_{i j}\right| H_{j} x_{i}(t)+\sum_{j=1}^{n}\left|v_{i j}\right| H_{j} \int_{-\infty}^{t} e^{\varepsilon(t-s)} K_{i j}(t-s) x_{j}(s) d s\right] \\
& +\frac{1}{2}(p-1)(p-2) \sum_{j=1}^{n} s_{i j} x_{i}(t)+(p-1) \sum_{j=1}^{n} s_{i j} x_{j}(t) \\
& +\frac{1}{2}(p-1)(p-2) \sum_{j=1}^{n} w_{i j} x_{i}(t)+(p-1) \sum_{j=1}^{n} w_{i j} e^{\varepsilon \tau_{i j}(t)} x_{j}\left(t-\tau_{i j}(t)\right) \\
\leq & A_{i}\left\{\left[-p b_{i} \frac{a_{i}}{A_{i}}+\frac{\varepsilon}{A_{i}}+(p-1)\left(\sum_{j=1}^{n}\left|c_{i j}\right| G_{j}+\sum_{j=1}^{n}\left|d_{i j}\right| F_{j}+\sum_{j=1}^{n}\left|v_{i j}\right| H_{j}\right.\right.\right. \\
& \left.\left.+\frac{p-2}{2 A_{i}} \sum_{j=1}^{n} s_{i j}+\frac{p-2}{2 A_{i}} \sum_{j=1}^{n} w_{i j}\right)\right] x_{i}(t)+\sum_{j=1}^{n}\left(\left|c_{i j}\right| G_{j}+\frac{p-1}{A_{i}} s_{i j}\right) x_{j}(t) \\
& +e^{\varepsilon \tau} \sum_{j=1}^{n}\left(\left|d_{i j}\right| F_{j}+\frac{p-1}{A_{i}} w_{i j}\right) x_{j}\left(t-\tau_{i j}(t)\right) \\
& \left.+\sum_{j=1}^{n}\left|v_{i j}\right| H_{j} \int_{-\infty}^{t} e^{\varepsilon(t-s)} K_{i j}(t-s) x_{j}(s) d s\right\}
\end{aligned}
$$

for $i=1,2, \cdots, n ; t_{k-1}<t<t_{k}, k=1,2, \cdots$. Further, we can get

$$
\begin{aligned}
D^{+}\left(E x_{i}(t)\right) \leq & A_{i}\left\{\left[-p b_{i} \frac{a_{i}}{A_{i}}+\frac{\varepsilon}{A_{i}}+(p-1)\left(\sum_{j=1}^{n}\left|c_{i j}\right| G_{j}+\sum_{j=1}^{n}\left|d_{i j}\right| F_{j}+\sum_{j=1}^{n}\left|v_{i j}\right| H_{j}\right.\right.\right. \\
& \left.\left.+\frac{p-2}{2 A_{i}} \sum_{j=1}^{n} s_{i j}+\frac{p-2}{2 A_{i}} \sum_{j=1}^{n} w_{i j}\right)\right] E x_{i}(t)+\sum_{j=1}^{n}\left(\left|c_{i j}\right| G_{j}+\frac{p-1}{A_{i}} s_{i j}\right) E x_{j}(t) \\
& +e^{\varepsilon \tau} \sum_{j=1}^{n}\left(\left|d_{i j}\right| F_{j}+\frac{p-1}{A_{i}} w_{i j}\right) E x_{j}\left(t-\tau_{i j}(t)\right) \\
& \left.+\sum_{j=1}^{n}\left|v_{i j}\right| H_{j} \int_{-\infty}^{t} e^{\varepsilon(t-s)} K_{i j}(t-s) E x_{j}(s) d s\right\}
\end{aligned}
$$

for $i=1,2, \cdots, n ; t_{k-1}<t<t_{k}, k=1,2, \cdots$.

Letting

$$
l_{0}=\frac{\left\|\phi-u^{*}\right\|_{L_{p}}^{p}}{\min _{1 \leq i \leq n}\left\{\xi_{i}\right\}}
$$

then when $s \in\left(-\infty, t_{0}\right]$, we have

$$
E x_{i}(s)=e^{\varepsilon\left(s-t_{0}\right)} E\left|y_{i}(s)\right|^{p} \leq E\left|y_{i}(s)\right|^{p}=E\left|\phi_{i}\left(s-t_{0}\right)-u_{i}^{*}\right|^{p} \leq\left\|\phi-u^{*}\right\|_{L_{p}}^{p} \leq \xi_{i} l_{0}, \quad i=1,2, \cdots, n .
$$

Let us now prove

$$
E x_{i}(t) \leq \xi_{i} l_{0}, \quad t_{0} \leq t<t_{1}, \quad i=1,2, \cdots, n .
$$


In fact, if (13) is not true, then there exist some $i_{0}$ and $t^{*} \in\left[t_{0}, t_{1}\right)$ such that

$$
E x_{i_{0}}\left(t^{*}\right)=\xi_{i_{0}} l_{0}, \quad D^{+} E x_{i_{0}}\left(t^{*}\right) \geq 0 \quad \text { and } \quad E x_{j}(t) \leq \xi_{j} l_{0}, \quad t \in\left(-\infty, t^{*}\right), \quad j=1,2, \cdots, n
$$

However, from (11), (14) and (H4), we get

$$
\begin{aligned}
D^{+}\left(E x_{i_{0}}\left(t^{*}\right)\right) \leq & A_{i_{0}}\left\{\left[-p b_{i_{0}} \frac{a_{i_{0}}}{A_{i_{0}}}+\frac{\varepsilon}{A_{i_{0}}}+(p-1)\left(\sum_{j=1}^{n}\left|c_{i_{0} j}\right| G_{j}+\sum_{j=1}^{n}\left|d_{i_{0} j}\right| F_{j}+\sum_{j=1}^{n}\left|v_{i_{0} j}\right| H_{j}\right.\right.\right. \\
& \left.\left.+\frac{p-2}{2 A_{i_{0}}} \sum_{j=1}^{n} s_{i_{0} j}+\frac{p-2}{2 A_{i_{0}}} \sum_{j=1}^{n} w_{i_{0} j}\right)\right] \xi_{i_{0}}+\sum_{j=1}^{n}\left[\left(\left|c_{i_{0} j}\right| G_{j}+\frac{p-1}{A_{i_{0}}} s_{i_{0} j}\right)\right. \\
& \left.\left.+e^{\varepsilon \tau}\left(\left|d_{i_{0} j}\right| F_{j}+\frac{p-1}{A_{i_{0}}} w_{i_{0} j}\right)+\left|v_{i_{0} j}\right| H_{j} r_{i_{0} j}(\varepsilon)\right] \xi_{j}\right\} l_{0} .
\end{aligned}
$$

It follows from (10) and (15) that

$$
D^{+}\left(E x_{i_{0}}\left(t^{*}\right)\right)<0
$$

and this is a contradiction. So (13) is true.

In the following, we will use the mathematical induction to prove that

$$
E x_{i}(t) \leq \xi_{i} l_{0}, \quad t_{k-1} \leq t<t_{k}, \quad i=1,2, \cdots, n,
$$

holds for $k=1,2, \cdots$.

When $k=1$, we know from (13) that (16) holds. Suppose that the inequalities

$$
E x_{i}(t) \leq \xi_{i} l_{0}, \quad t_{k-1} \leq t<t_{k}, \quad i=1,2, \cdots, n,
$$

hold for $k=1,2, \cdots, m$.

From condition (ii) of this theorem, we have

$$
\left|u_{i}\left(t_{k}\right)-u_{i}^{*}\right|=\left|u_{i}\left(t_{k}^{-}\right)+J_{k}\left(u_{i}\left(t_{k}^{-}\right)\right)-u_{i}^{*}\right|=\left|1-\gamma_{i k}\right|\left|\left(u_{i}\left(t_{k}^{-}\right)-u_{i}^{*}\right)\right| \leq\left|u_{i}\left(t_{k}^{-}\right)-u_{i}^{*}\right|
$$

for $i=1,2, \cdots, n ; k=1,2, \cdots$. Hence

$$
x_{i}\left(t_{k}\right) \leq x_{i}\left(t_{k}^{-}\right), \quad i=1,2, \cdots, n ; \quad k=1,2, \cdots
$$

Further, we can get

$$
E x_{i}\left(t_{k}\right) \leq E x_{i}\left(t_{k}^{-}\right), \quad i=1,2, \cdots, n ; \quad k=1,2, \cdots .
$$

It follows from (17) and (18) that

$$
E x_{i}\left(t_{m}\right) \leq E x_{i}\left(t_{m}^{-}\right)<\xi_{i} l_{0} . \quad i=1,2, \cdots, n .
$$

This, together with both (12), (17) and (19), lead to

$$
E x_{i}(t) \leq \xi_{i} l_{0}, \quad t \in\left(-\infty, t_{m}\right], \quad i=1,2, \cdots, n,
$$

It is similar to the proof of (13), we can prove that

$$
E x_{i}(t) \leq \xi_{i} l_{0}, \quad t_{m} \leq t<t_{m+1}, \quad i=1,2, \cdots, n .
$$


To this end, by the mathematical induction, we can conclude that (16) holds. Thus

$$
E\left|u_{i}(t)-u^{*}\right|^{p} \leq \xi_{i} l_{0} e^{-\varepsilon\left(t-t_{0}\right)}, \quad t \geq t_{0}, \quad i=1,2, \cdots, n .
$$

So

$$
E\left\|u(t)-u^{*}\right\|^{p} \leq M\left\|\phi-u^{*}\right\|_{L_{p}}^{p} e^{-\varepsilon\left(t-t_{0}\right)}, \quad t \geq t_{0}
$$

where $M=\sum_{i=1}^{n} \xi_{i} / \min _{1 \leq i \leq n}\left\{\xi_{i}\right\} \geq 1$. This means that the unique equilibrium point $u^{*}$ of model (1) is globally exponentially $p$-stable, and the exponential convergence rate equals $\varepsilon$ from (10). The proof is completed.

Remark 2. In this paper, the proposed method, which does not make use of the Lyapunov functional, is shown to be simple yet effective for analyzing the stability of impulsive or stochastic neural networks with variable and/or distributed delays.

Remark 3. In $[28,33,34]$, the authors have dealt with the robust stability of uncertain stochastic neural networks with delays by employing Lyapunov functional. Using the method of this paper, we can also deal with the robust stability of uncertain system (1) in a fairly straightforward way. For example, when $c_{i j} \in\left[\underline{c}_{i j}, \bar{c}_{i j}\right]$, $d_{i j} \in\left[\underline{d}_{i j}, \bar{d}_{i j}\right], v_{i j} \in\left[\underline{v}_{i j}, \bar{v}_{i j}\right]$, let

$$
\begin{aligned}
c_{i j}^{(0)} & =\frac{1}{2}\left(\bar{c}_{i j}+\underline{c}_{i j}\right), & c_{i j}^{(1)} & =\frac{1}{2}\left(\bar{c}_{i j}-\underline{c}_{i j}\right), \\
d_{i j}^{(0)} & =\frac{1}{2}\left(\bar{d}_{i j}+\underline{d}_{i j}\right), & d_{i j}^{(1)} & =\frac{1}{2}\left(\bar{d}_{i j}-\underline{d}_{i j}\right), \\
v_{i j}^{(0)} & =\frac{1}{2}\left(\bar{v}_{i j}+\underline{v}_{i j}\right), & v_{i j}^{(1)} & =\frac{1}{2}\left(\bar{v}_{i j}-\underline{v}_{i j}\right) .
\end{aligned}
$$

Noting

$$
\left|c_{i j}\right| \leq\left|c_{i j}^{(0)}\right|+c_{i j}^{(1)}, \quad\left|d_{i j}\right| \leq\left|d_{i j}^{(0)}\right|+d_{i j}^{(1)}, \quad\left|v_{i j}\right| \leq\left|v_{i j}^{(0)}\right|+v_{i j}^{(1)},
$$

similar to the proof theorem 1, it is easy to prove the following corollary.

Corollary 1: Under assumptions $(\mathbf{H} 1)-(\mathbf{H} 5)$, if there exists a positive constant $p \geq 2$ such that $-(Q+T)$ is an $M$-matrix, where

$$
\begin{gathered}
Q=\left(q_{i j}\right)_{n \times n}, \quad q_{i j}=\left(\left|c_{i j}^{(0)}\right|+c_{i j}^{(1)}\right) G_{j}+\frac{p-1}{A_{i}} s_{i j}, \quad i \neq j, \\
q_{i i}=-p b_{i} \frac{a_{i}}{A_{i}}+(p-1)\left(\sum_{j=1}^{n}\left(\left|c_{i j}^{(0)}\right|+c_{i j}^{(1)}\right) G_{j}+\sum_{j=1}^{n}\left(\left|d_{i j}^{(0)}\right|+d_{i j}^{(1)}\right) F_{j}+\sum_{j=1}^{n}\left(\left|v_{i j}^{(0)}\right|+v_{i j}^{(1)}\right) H_{j}\right. \\
\left.+\frac{p-2}{2 A_{i}} \sum_{j=1}^{n} s_{i j}+\frac{p-2}{2 A_{i}} \sum_{j=1}^{n} w_{i j}\right)+\left(\left|c_{i i}^{(0)}\right|+c_{i i}^{(1)}\right) G_{i}+\frac{p-1}{A_{i}} s_{i i}, \\
T=\left(t_{i j}\right)_{n \times n}, \quad t_{i j}=\left(\left|d_{i j}^{(0)}\right|+d_{i j}^{(1)}\right) F_{j}+\frac{p-1}{A_{i}} w_{i j}+\left(\left|v_{i j}^{(0)}\right|+v_{i j}^{(1)}\right) H_{j},
\end{gathered}
$$

then model (4) has a unique equilibrium point $\left(u_{1}^{*}, u_{2}^{*}, \cdots, u_{n}^{*}\right)^{T}$. Further suppose

(i) $\sigma_{i j}\left(u_{j}^{*}, u_{j}^{*}\right)=0, i, j=1,2, \cdots, n$.

(ii) $J_{k}\left(u_{i}\left(t_{k}\right)\right)=-\gamma_{i k}\left(u_{i}\left(t_{k}^{-}\right)-u_{i}^{*}\right), \quad 0<\gamma_{i k}<2, \quad i=1,2, \cdots, n ; \quad k=1,2, \cdots$.

Then $\left(u_{1}^{*}, u_{2}^{*}, \cdots, u_{n}^{*}\right)^{T}$ is a unique globally exponentially $p$-stable equilibrium point of model (1).

Remark 4. Recently, the linear matrix inequality (LMI) approach has been popular in dealing with the stability of the delayed neural networks, and the obtained criteria by using the LMI approach are in general less conservative than the criteria by using the $M$-matrix approach, for example, see [9,25,28,29]. Unfortunately, 
in $[9,25,28,29]$, the active functions are assumed to be bounded in order to guarantee the existence of the equilibrium point of the considered neural networks. When the active functions are indeed unbounded, the stability criteria provided in $[9,25,28,29]$ may be difficult to apply due to the questionable existence of the equilibrium point.

Remark 5. From Theorem 1 and Corollary 1, we can see that the stability of model (1) are mainly affected by the parameters of the continuous part of model (1).

Remark 6. In [35-37], the authors have considered the discrete-time systems with time-varying state delay and obtained several new results of stability by the LMI approach. We would like to point out that it is possible to generalize our main results to discrete-time systems. The results will appear in the near future.

\section{EXAMPLE}

Example 1. Consider the following model

$$
\left\{\begin{aligned}
\frac{d u_{1}(t)}{d t}=- & \left(2+\cos u_{1}(t)\right)\left[18 u_{1}(t)-0.1 g_{1}\left(u_{1}(t)\right)+0.7 g_{2}\left(u_{2}(t)\right)\right. \\
& -f_{1}\left(u_{1}\left(t-\tau_{11}(t)\right)\right)-0.8 f_{2}\left(u_{2}\left(t-\tau_{12}(t)\right)\right) \\
& \left.-2 \int_{-\infty}^{t} K_{11}(t-s) h_{1}(s) d s+\int_{-\infty}^{t} K_{12}(t-s) h_{2}(s) d s\right] \\
& +\sigma_{11}\left(u_{1}(t), u_{1}\left(t-\tau_{11}(t)\right)\right) d \omega_{1}+\sigma_{12}\left(u_{2}(t), u_{2}\left(t-\tau_{12}(t)\right)\right) d \omega_{2}, \quad t \neq t_{k}, \\
\frac{d u_{2}(t)}{d t}=- & \left(3-\sin u_{2}(t)\right)\left[15 u_{2}(t)+g_{1}\left(u_{1}(t)\right)+0.5 g_{2}\left(u_{2}(t)\right)\right. \\
& -0.9 f_{1}\left(u_{1}\left(t-\tau_{21}(t)\right)\right)-2 f_{2}\left(u_{2}\left(t-\tau_{22}(t)\right)\right) \\
& \left.-\int_{-\infty}^{t} K_{11}(t-s) h_{1}(s) d s+2 \int_{-\infty}^{t} K_{12}(t-s) h_{2}(s) d s\right] \\
& +\sigma_{21}\left(u_{1}(t), u_{1}\left(t-\tau_{21}(t)\right)\right) d \omega_{1}+\sigma_{22}\left(u_{2}(t), u_{2}\left(t-\tau_{22}(t)\right)\right) d \omega_{2}, \quad t \neq t_{k}, \\
\Delta u_{1}\left(t_{k}\right)= & -(1+0.5 \sin (1+k)) u_{1}\left(t^{-}\right), \\
\Delta u_{2}\left(t_{k}\right)= & -\left(1+0.8 \cos \left(2 k^{3}\right)\right) u_{1}\left(t^{-}\right)
\end{aligned}\right.
$$

where $t_{0}=0, t_{k}=t_{k-1}+0.5 k, k=1,2, \cdots$, and

$$
\begin{gathered}
g_{i}(x)=f_{i}(x)=h_{i}(x)=x, \quad \tau_{i j}(t)=0.2|\cos t|+0.1, \quad K_{i j}(t)=t e^{-t}, \quad i, j=1,2, \\
\sigma_{11}(x, y)=0.1 x-0.2 y, \quad \sigma_{12}(x, y)=0.2 x+0.3 y, \\
\sigma_{21}(x, y)=0.5 x+0.4 y, \quad \sigma_{22}(x, y)=0.3 x+0.1 y .
\end{gathered}
$$

Obviously, model (23) satisfies assumptions (H1)-(H4) with

$$
a_{1}=1, \quad A_{1}=3, \quad a_{2}=2, \quad A_{2}=4, \quad b_{1}=18, \quad b_{2}=15, \quad F_{i}=G_{i}=H_{i}=1, \quad i=1,2 .
$$

It can be easily checked that assumption (H5) is also satisfied with

$$
\begin{gathered}
s_{11}=0.02, \quad s_{12}=0.08, \quad s_{21}=0.5, \quad s_{22}=0.18, \\
w_{11}=0.08, \quad w_{12}=0.18, \quad w_{21}=0.32, \quad w_{22}=0.02 .
\end{gathered}
$$

Taking $p=4$, it is easy to compute

$$
Q=\left(\begin{array}{cc}
-6.72 & 0.78 \\
1.375 & -6.4
\end{array}\right), \quad T=\left(\begin{array}{cc}
3.08 & 1.26 \\
2.14 & 4.015
\end{array}\right),
$$


and

$$
-(Q+T)=\left(\begin{array}{cc}
3.64 & -2.04 \\
-3.515 & 2.385
\end{array}\right)
$$

is an $M$-matrix. On the anther hand, one can verify that $(0,0)^{T}$ is an equilibrium point of model (23).

Clearly, all conditions of Theorem 1 are satisfied. From Theorem 1, we know that $(0,0)^{T}$ is a unique globally exponentially 4-stable equilibrium point of model (23). From (10), we can estimate that the exponential convergence rate index is equal to 0.0145 .

\section{Conclusions}

In this paper, the problem on stability analysis has been investigated for a class of impulsive stochastic Cohen-Grossberg neural networks with both time-varying and infinite distributed delays. A sufficient condition to ensure the existence, uniqueness, and exponential $p$-stability of equilibrium point for the addressed neural network has been obtained by employing a combination of the $M$-matrix theory and stochastic analysis technique. The proposed method, which does not make use of the Lyapunov functional, has been shown to be simple yet effective for analyzing the stability of impulsive or stochastic neural networks with variable and/or distributed delays. We have then extended our main results to the case where the parameters contain interval uncertainties. The exponential convergence rate index can be estimated that is dependent on the system parameters. An example has been given to show the effectiveness of the obtained results.

\section{AcKnowledgements}

The authors would like to thank the reviewers and the editor for their valuable suggestions and comments which have led to a much improved paper.

\section{REFERENCES}

[1] M.A. Cohen, S. Grossberg, Absolute stability of global pattern formation and parallel memory storage by competitive neural networks, IEEE Transactions on Systems, Man, and Cybernetics 13(5)(1983) 815-826.

[2] J.L. Liang, J.D. Cao, Global output convergence of recurrent neural networks with distributed delays, Nonlinear Analysis: Real World Applications 8(1)(2007) 187-197.

[3] H. Ye, A.N. Michel, K.N. Wang, Qualitative analysis of Cohen-Grossberg neural networks with multiple delays. Physical Review E 51(3)(1995) 2611-2618.

[4] L. Wang, X.F. Zou, Harmless delays in Cohen-Grossberg neural networks, Physica D 170(2)(2002) 162-173.

[5] W.L. Lu, T.P. Chen, New conditions on global stability of Cohen-Grossberg neural networks, Neural Computation 15(5) (2003) 1173-1189.

[6] T.P. Chen, L.B. Rong, Delay-independent stability analysis of Cohen-Grossberg neural networks, Physics Letters A 317(56)(2003) 436-449.

[7] T.P. Chen, L.B. Rong, Robust global exponential stability of Cohen-Grossberg neural networks with time delays, IEEE Transactions on Neural Networks 15(1)(2004) 203-206.

[8] J.D. Cao, J.L. Liang, Boundedness and stability for Cohen-Grossberg neural network with time-varying delays, Journal of Mathematical Analysis and Applications 296(2)(2004) 665-685.

[9] J.D. Cao, X.L. Li, Stability in delayed Cohen-Grossberg neural networks: LMI optimization approach, Physica D 212(12)(2005) 54-65.

[10] S. Arik, Z. Orman, Global stability analysis of Cohen-Grossberg neural networks with time varying delays, Physics Letters A 341(5-6)(2005) 410-421.

[11] K. Yuan, J.D. Cao. An analysis of global asymptotic stability of delayed Cohen-Grossberg neural networks via nonsmooth analysis, IEEE Transactions on Circuits and Systems I 52(9)(2005) 1854-1861.

[12] K.N. Lu, D.Y. Xu, Z.C. Yang, Global attraction and stability for Cohen-Grossberg neural networks with delays, Neural Networks 19(10)(2006) 1358-1549. 
[13] Q.K. Song, J.D. Cao, Stability analysis of Cohen-Grossberg neural network with both time-varying and continuously distributed delays, Journal of Computational and Applied Mathematics 197(1)(2006) 188-203.

[14] Z.H. Guan, G.R. Chen, On delayed impulsive Hopfield neural networks, Neural Networks 12(2)(1999) 273-280.

[15] K. Gopalsamy, Stability of artificial neural networks with impulses, Applied Mathematics and Computation 154(3)(2004) 783-813.

[16] Y. Zhang, J.T. Sun, Stability of impulsive neural networks with time delays, Physics Letters A 348(1-2)(2005) 44-50.

[17] D.Y. Xu, W. Zhu, S.J. Long, Global exponential stability of impulsive integro-differential equation, Nonlinear Analysis 64(12)(2006) 2805-2816.

[18] D.W.C. Ho, J.L. Liang, J. Lam, Global exponential stability of impulsive high-order BAM neural networks with time-varying delays, Neural Networks 19(10)(2006) 1581-1590.

[19] Z. Chen, J. Ruan, Global dynamic analysis of general Cohen-Grossberg neural networks with impulse, Chaos, Solitons and Fractals 32(5)(2007) 1830-1837.

[20] Q.K. Song, J.Y. Zhang, Global exponential stability of impulsive Cohen-Grossberg neural network with time-varying delays, Nonlinear Analysis: Real World Applications (2006) doi:10.1016/j.nonrwa.2006.11.015.

[21] Z.G. Yang, D.Y. Xu, L. Xiang, Exponential p-stability of impulsive stochastic differential equations with delays, Physics Letters A 359(2)(2006) 129-137.

[22] H.J. Wu, J.T. Sun, p-Moment stability of stochastic differential equations with impulsive jump and Markovian switching, Automatica 42(10)(2006) 1753-1759.

[23] Z.W. Gao, D.W.C. Ho, State/noise estimator for descriptor systems with application to sensor fault diagnosis, IEEE Transactions on Signal Processing 54(4)(2006) 1316-1326.

[24] H.Y. Zhao, N. Ding, Dynamic analysis of stochastic Cohen-Grossberg neural networks with time delays, Applied Mathematics and Computation 183(1)(2006) 464-470.

[25] Y. Liu, Z. Wang, X. Liu, On global exponential stability of generalized stochastic neural networks with mixed time-delays, Neurocomputing 70(1-3)(2006) 314-326.

[26] Y. Liu, Z. Wang, X. Liu, Stability criteria for periodic neural networks with discrete and distributed delays, Nonlinear Dynamics, 49(1-2)(2007) 93-103.

[27] Y. Liu, Z. Wang, X. Liu, Design of exponential state estimators for neural networks with mixed time delays, Physics Letters A, 364(5)(2007) 401-412.

[28] Z. Wang, Y. Liu, K. Fraser, X. Liu, Stochastic stability of uncertain Hopfield neural networks with discrete and distributed delays, Physics Letters A 354(4)(2006) 288-297.

[29] Z. Wang, Y. Liu, M. Li, X. Liu, Stability analysis for stochastic Cohen-Grossberg neural networks with mixed time delays, IEEE Transactions on Neural Networks 17(2006) 814-820.

[30] Z. Wang, Y. Liu, X. Liu, On global asymptotic stability of neural networks with discrete and distributed delays, Physics Letters A 345(4-6)(2005) 299-308.

[31] Z. Wang, H. Unbehauen, Robust $H_{2} / H_{\infty}$ state estimation for systems with error variance constraints: the continuous-time case, IEEE Transactions on Automatic Control, 44(5)(1999), 1061-1065.

[32] Z. Wang, Z. Guo, H. Unbehauen, Robust $H_{2} / H_{\infty}$ state estimation for discrete-time systems with error variance constraints, IEEE Transactions on Automatic Control, 42(10)(1997), 1431-1435.

[33] H. Huang, G. Feng, Delay-dependent stability for uncertain stochastic neural networks with time-varying delay, Physica A 381(2007) 93-103.

[34] J.H. Zhang, P. Shi, J.Q. Qiu, Novel robust stability criteria for uncertain stochastic Hopfield neural networks with timevarying delays, Nonlinear Analysis: Real World Applications 8(4)(2007) 1349-1357.

[35] H. Gao, T. Chen, New results on stability of discrete-time systems with time-varying state delay, IEEE Transations on automatic control 52(2)(2007) 328-334.

[36] H. Gao, J. Lam, G. Chen, New criteria for synchronization stability of general complex dynamical networks with coupling delays, Physics Letters A 360(2)(2006) 263-273.

[37] H. Gao, P. Shi, J. Wang, Parameter-dependent robust stability of uncertain time-delay systems, J. Computational and Applied Mathematics 206(1)(2007) 366-373. 